\title{
La crisis de la seguridad pública y los resultados electorales en el retorno del PRI a Nuevo León. Un análisis retrospectivo
}

\author{
Laura Nelly Medellín Mendoza*
}

Resumen: en este artículo se revisa la problemática reciente de la seguridad en la agenda pública de Nuevo León. Se destaca el efecto en los resultados electorales para el partido gobernante, tanto en elecciones intermedias como en la sucesión por la gubernatura. En ese sentido, el periodo de gobierno de 2003 a 2009 tuvo que enfrentar los altos índices delictivos con promulgación de leyes y políticas públicas, para marcar una reestructuración institucional en esta área. Se encontró evidencia de que el partido gobernante perdió espacios en los comicios intermedios en un contexto de gran inseguridad, pero esto no repercutió en la sucesión por la gubernatura, aunque los índices de homicidios por narcotráfico y la percepción de inseguridad ciudadana fueron altos hasta el término de la administración.

Palabras clave: Nuevo León; partido gobernante; elecciones; seguridad pública; índices delictivos; percepción de inseguridad. 
Abstract: this article reviews the recent public security problem on Nuevo Leon's public agenda. It highlights how this problem has potentially affected the governing party's political capital by examining midterm and gubernatorial election results. During the period of study, 2003-2009, the government tackled high crime rates by developing specific laws and public policies, indicating an institutional restructuring in this area. We find evidence that in a context of high public insecurity, the governing party lost legislative and mayoral seats during the midterms, yet the governor's race was not affected despite the fact that high murder rates connected to drug trafficking and high perceptions of insecurity persisted throughout the governing party's term in office.

Key words: Nuevo León; ruling party; elections; public security; crime rates; perception of insecurity.

\section{Introducción}

Una condición fundamental para la legitimidad del Estado es proporcionar el bien político de la seguridad pública, de acuerdo con un modelo contractualista hobbesiano. El problema de investigación aquí es indagar si una crisis severa de seguridad pública puede incidir en un gobierno, que es responsable de las políticas de Estado, para que el electorado pueda darle o retirarle su voto para seguir gobernando.

Para lo anterior se ubicó el sexenio del gobernador de Nuevo León, Natividad González Parás (2003-2009), quien representó el regreso del Partido Revolucionario Institucional (PRI), después de un periodo de alternancia en el que rigió el Partido Acción Nacional (PAN) (1997-2003). En esa administración se dispararon los índices delictivos, lo que tuvo un efecto negativo en la percepción ciudadana de la seguridad. Las hipótesis centrales son: a) al gobierno priista no le perjudicó sustancialmente el tema de la seguridad pública en la sucesión por la gubernatura de 2009, a pesar de que perdió espacios 
de poder en las elecciones intermedias, en las que sí existió esta correlación; b) la producción de leyes y políticas en seguridad pública tuvo un efecto parcial en la disminución de los índices delictivos, a excepción de los homicidios relacionados con el narcotráfico y la percepción de inseguridad, ya que ambos continuaron incrementándose.

La metodología fue medir la dimensión del partido gobernante para lo que se tuvo en cuenta, como variable principal, los resultados en los comicios intermedios y las elecciones siguientes de gobernador. Los indicadores son el número de diputados electos y de alcaldías ganadas por el priismo, así como los resultados de la elección por la gubernatura; los resultados de los comicios de 2003 fue la variable antecedente para estos mismos indicadores. Después se midió la crisis en materia de seguridad pública, cuya variable principal fue la incidencia de delitos de alto impacto; sus indicadores se refirieron a la tasa de homicidios dolosos (y los relacionados con narcotráfico), los robos, las extorsiones y los secuestros comparables con el sexenio anterior. Las respuestas gubernamentales constituyeron la siguiente variable que se dividió en tres indicadores principales: la normatividad jurídica, el desarrollo institucional y las instancias de participación ciudadana. Por último se utilizó la variable opinión de la ciudadanía sobre el tema de la seguridad, sólo se empleó el indicador de encuestas de percepción de inseguridad.

\section{La producción del "bien político" de la seguridad pública y la decisión del voto}

En teoría, la responsabilidad primaria del Estado es asegurar el orden público (Peña 2011, 27; Ramírez 2010, 120) y la convivencia pacífica de los ciudadanos; por tanto, la seguridad pública es una condición fundante. En contrapartida, la inseguridad se vería como una debilidad estructurante del Estado. Para justificar teóricamente lo anterior, se revisó el marco de discusión de la teoría contractualista, uno de cuyos puntos fundamentales tiene que ver con la conceptualización que se tiene del Estado, entendida como un conjunto de insti- 
tuciones que refieren las estructuras legales y de control que expresan el contrato social, que se entiende como:

“[...] la representación de las relaciones sociales que estructuran a un determinado grupo de personas en un tiempo y en un espacio dado $[\ldots]$ a través de la determinación de los derechos y responsabilidades de cada grupo. Entonces, el contrato social es la manifestación del Estado en tanto que representa la voluntad de los ciudadanos para vivir bajo el Estado de derecho" (Tedesco 2007, 8).

Ahora bien, si se parte de la visión contractualista en perspectiva hobbesiana, se entendería que los ciudadanos ceden parte de sus libertades para otorgárselas al Estado, con el fin de que les garantice seguridad, frente al miedo de vivir en "guerra permanente unos con otros”. Como bien señala Ibarra $(2009,21)$, El leviatán de Hobbes protege intereses, no derechos. De acuerdo con Hobbes, el miedo es el acto instintivo que somete al individuo al Estado, porque teme por su vida (Robin 2009, 68).

Sin embargo, en un marco constitucional democrático la garantía de los derechos humanos estaría protegida por el mismo Estado. Hay que ser preciso en esta distinción, porque se conocen las críticas al modelo hobbesiano, por su tendencia a justificar un Estado totalitario (Ramírez 2010), o como señala Yates (2014) lo que desde El leviatán se advierte como el "derecho a castigar". Aquí se destaca que la seguridad pública es una necesidad de convivencia societal ineludible que, cuando se pierde, el Estado soberano también tiene la responsabilidad primaria de garantizar.

En estas definiciones, la responsabilidad es de una elite gobernante determinada, que necesita resultados eficaces para lograr su legitimidad electoral y permanencia en los cargos públicos. Estas dos últimas variables están íntimamente relacionadas con las obligaciones concebibles de producir bienes políticos, que el Estado proporciona a su población, así como sus responsabilidades de proveer las garantías constitucionales para el bienestar social de los ciudadanos; en este análisis, la dimensión preponderante es la seguridad pública (Rotberg 2007, 157-158). Es evidente que las acciones de un gobierno se pueden analizar a partir de más variables, como la provisión del 
empleo público, de servicios educativos, infraestructura hospitalaria y combate a la pobreza, entre otras. Sin embargo, esta decisión metodológica no es aleatoria, sino que corresponde a la demanda principal en la agenda pública en este periodo. Ante la escalada imparable de la violencia, los nuevoleoneses demandaron seguridad principalmente, con marchas y manifestaciones públicas (Contreras y Zamora 2013). Otros estudios locales también señalaban que la preocupación primordial era la inseguridad, relacionada con la irrupción del narcotráfico en los espacios públicos (Centro de Investigaciones Económicas 2010, 34; Cantú y Garza 2009, 76).

Existen diferentes teorías del comportamiento electoral que explicarían las decisiones de electores respecto a su sentido de votación. De forma esquemática, las principales corrientes son las escuelas de Columbia, Michigan y la de elección racional (Downs 1957; Berlanga yVázquez 2009; Koschick 2004). La primera enfatiza que la identidad y el comportamiento electoral de los votantes no cambian necesariamente por la influencia de las campañas electorales, ya que hay una determinación dada su pertenencia de clase (Lazarfeld et al. 1969). La escuela de Michigan tiene un perfil psicosocial, ya que señala que los votantes están en mayor medida influidos por su identidad política-partidaria, la imagen de los candidatos y los temas de campaña (Campbell et al. 1960). Según la tercera corriente, los votantes actúan con base en un análisis de costo-beneficio para maximizar su interés individual (Downs 1957).

Hasta el momento sólo hay un estudio que analiza tres perfiles básicos del elector nuevoleonés: el partidista, el personalista y el inconforme (Cantú y Garza 2009). Sin embargo, aunque el presente trabajo está orientado a analizar los resultados de los comicios, no se hace una revisión minuciosa del comportamiento electoral. Lo que interesa es destacar cómo una crisis grave influye en materia de seguridad pública en la permanencia del partido en el gobierno. Se infiere el asunto de la seguridad pública como una variable contextual relevante por los indicadores de delitos de alto impacto a la alza y por la agenda setting (McCombs 1972), posicionada en la opinión pública, sobre todo a mitad del sexenio de González Parás. La percepción del riesgo del entorno, - de acuerdo con el enfoque de la escuela de Michigan-, se vuelve un factor importante en la decisión personal 
del votante (Blais 2006). La percepción humana puede interpretarse como la recepción de un tipo de realidad que rodea al sujeto, ya que la presencia de temor ante la posibilidad de sufrir un delito tiene un efecto en la rutinización de actividades cotidianas, que éste debe modificar para sentirse seguro. El delito, como lo sugiere Garland (2001, 255), cada vez más forma parte de la vida cotidiana, del habitus de las grandes ciudades.

Jiménez (2005) y Alcocer y Vásquez (2005) coinciden en señalar que son varios los factores que influyen en la percepción sobre la inseguridad, por ejemplo la sobreexposición en los medios de comunicación de los fenómenos de violencia, pero también la desconfianza de la ciudadanía en "los controles institucionales y la sensación de que hay un recrudecimiento de la corrupción” (Jiménez 2005, 331).

A partir de la problemática de inseguridad que se vivió en el estado se pueden inferir dos elementos importantes, que la elite gobernante priista tuvo que resolver: a) la reestructuración de la seguridad pública desde el inicio del gobierno, en 2003, debido al diagnóstico de inseguridad y b) las cifras de delitos del fuero común y los relacionados con el crimen organizado a la alza, y que tienen un efecto negativo en la percepción de inseguridad ciudadana.

\section{Del "orden y el progreso"}

a la tierra de la inseguridad y la violencia

Nuevo León cumplió por décadas, desde finales del siglo XIX, con la máxima positivista de orden y progreso. Las luchas ideológicas, lejos de poner en quiebre la estabilidad social, ${ }^{1}$ la entidad fue el escenario natural para la producción y circulación de mercancías por su condición de frontera con Estados Unidos. Este clima agradable para la expansión de fábricas desde esta época, como lo señalan los historiadores Cavazos y Ortega (2010, 211-217), fue gracias a una consolidada vocación industrial como referencia de identidad cultural simbólica de apego "al valor del trabajo" (Rojas 2006).

1 Salvo en los procesos históricos de lucha armada, como sería el independentista de 1810 y la revolución, en 1910. 
La estabilidad política del régimen, por su perfil de corte autoritario, al igual que en otros estados de la república, generó una serie de condiciones para que los procesos electorales no fueran razón de encarnecidas movilizaciones sociales, salvo el fraude electoral de 1985. La alternancia política de 1997 llegó debido a la fuerte presencia opositora del PAN, así como a las reformas electorales producidas un año antes, lo que generó una mayor confianza en la ciudadanización del órgano electoral.

Las elecciones de 2003 se distinguieron por el retorno del PRI a la gubernatura gracias a una combinación de factores, entre los que destacan la incapacidad de la administración panista (1997-2003) para gobernar con resultados democráticos (Medellín 2011), y a la campaña de posicionamiento de Natividad González Parás como representante de un nuevo PRI renovado y ciudadanizado (González 2012).

Educado en la Universidad de París, con doctorado en ciencia política, tenía las más altas cartas credenciales también para la actividad política. Su paso por diversos cargos centrales en la burocracia federal, desde finales de los años setenta, así como su experiencia como legislador y senador, desde los años noventa, lo habían colocado como un liderazgo clave en el ámbito local para contender por la gubernatura por el PRI, frente a la anterior elite dominante priista local de estirpe salinista que había salido "por la puerta de atrás", con la renuncia obligada del gobernador Sócrates Rizzo, ante la caída del proyecto salinista en el gobierno de Ernesto Zedillo. No obstante, su derrota electoral en 1997, como candidato priista, le permitió reorganizarse dentro de su elite dominante del partido para ganar en 2003. Cabe destacar que en esta elección, como en las que siguieron, el PRI formó una coalición con partidos minoritarios, sin que esto significara un compromiso de incluirlos en los espacios de gobierno en caso de resultar ganador. Por tanto, el PRI no formó una coalición, ya que no los incluyó en forma alguna de representación.

En la figura 1 se observa la diferencia entre González Parás y el candidato del PAN, Mauricio Fernández Garza, que fue de 22.86 puntos porcentuales: 56.66 para la AC y 33.8 para el PAN, lo que representó una diferencia de más de 350 mil votos entre el primer y segundo lugar (824 mil frente a $491 \mathrm{mil}$ ). 


\section{Figura 1}

\section{Votación por partido político o coalición} para elección de gobernador en Nuevo León, 2003

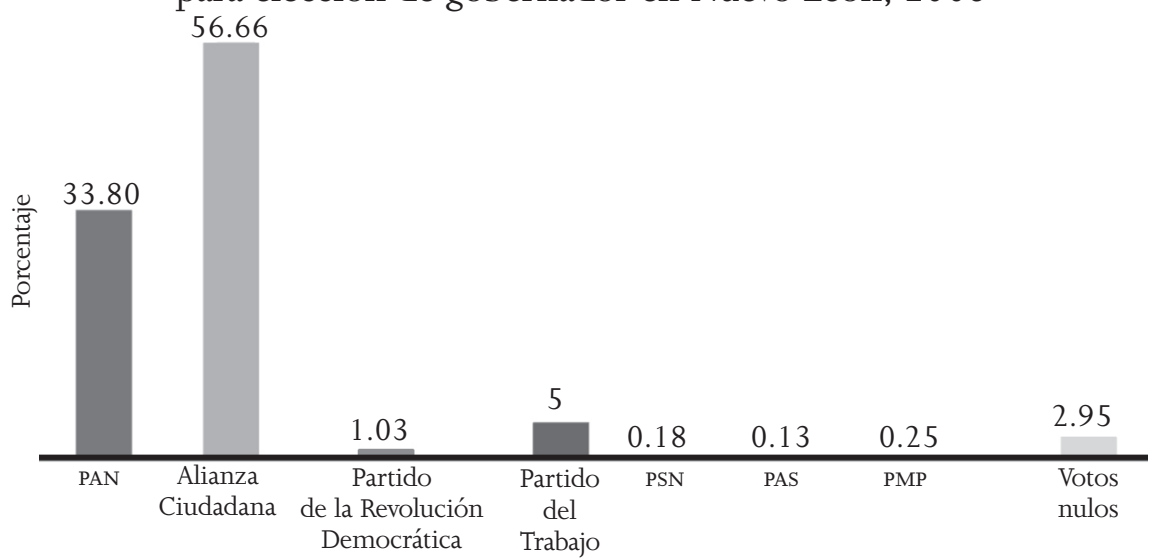

Nota: la coalición Alianza Ciudadana (AC) estuvo formada por el PRI, los partidos Verde Ecologista de México (PVEM), Fuerza Ciudadana (PFC) y el Liberal Mexicano (PLM). El Partido de la Sociedad Nacionalista (PNS), el Partido Alianza Social (PAS) y el Partido México Posible (PMP) perdieron su registro. Fuente: elaboración propia, con información de la Comisión Estatal Electoral (CEE 2004, 97-98).

Figura 2

Votación por partido político o coalición

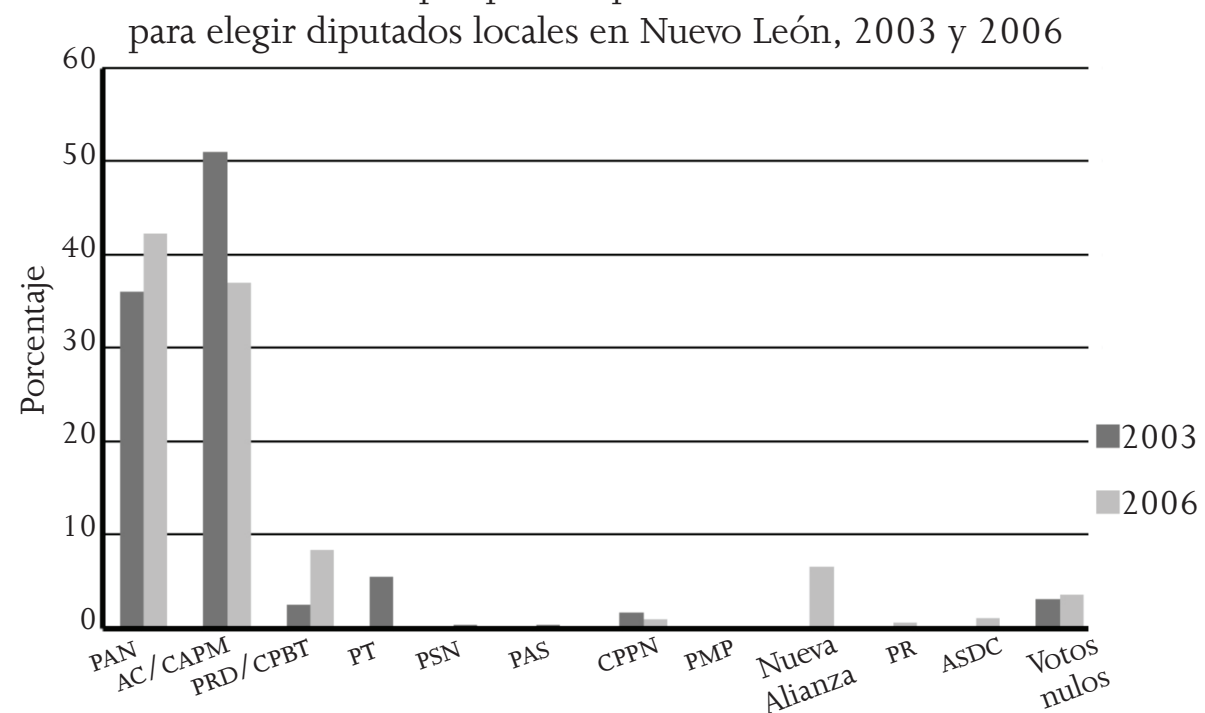

Nota: la coalición AC estuvo formada por PRI, PVEM, PFC y el PLM. El PSN, el PAS y el PMP perdieron su registro. En 2006, el PRI contendió con la Coalición Alianza por México (CAPM), que integró con el PVEM. La Coalición por el Bien de Todos (CPBT) estuvo formada por el PRD y el PT. La Convergencia Partido Político Nacional (CPPN), Nueva Alianza, el Partido Republicano (PR) y Alianza Social Demócrata (ASDC) no tuvieron alianza. Los dos últimos perdieron su registro en las siguientes elecciones. Fuente: elaboración propia, con información de la CEE $(2007,187 ; 2004,99)$. 
En las elecciones para el Legislativo en 2003, el PRI con su coalición AC mantuvo una victoria de 51 por ciento de la votación en comparación con el PAN, su principal opositor, que alcanzó 36.1 (véase figura 2). Sin embargo, en los comicios intermedios del sexenio de González Parás, en 2006, el PRI tuvo un descenso en las votaciones; quedó en segundo lugar, con la CAPM en el Congreso, con 36.9 por ciento, perdió su mayoría relativa frente al PAN, con 51.

\section{Figura 3}

Comparativo de diputados electos para el Congreso del estado por partido político o coalición en las elecciones locales, 2003 y 2006

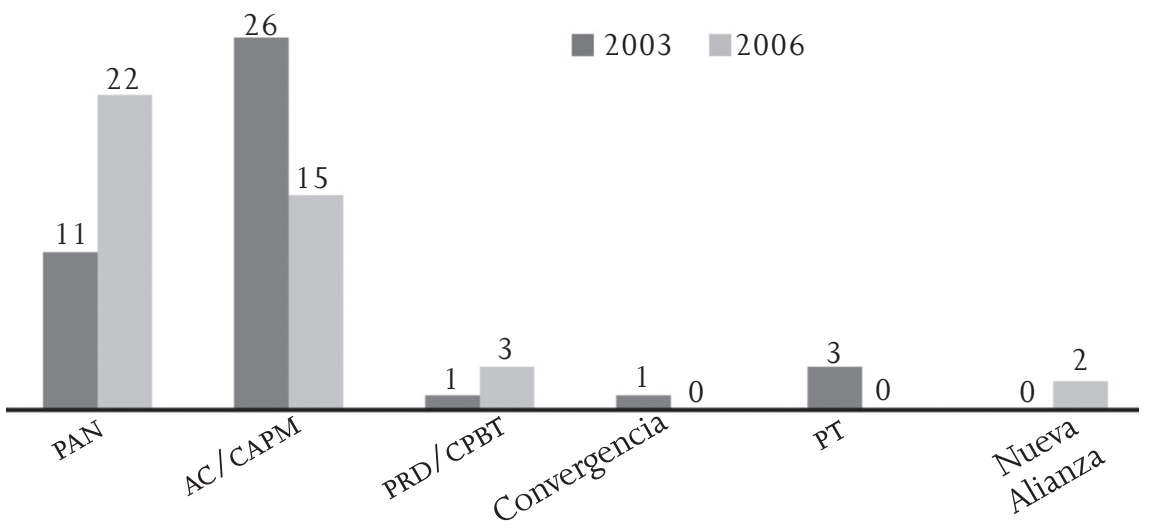

Nota: en 2003, la coalición AC estuvo formada por PRI, PVEM, PFC y PLM. En 2006 estuvo en la denominada CAPM, integrada por el PRI y el PVEM. La CBPT la conformaron el PRD y el PT. En las coaliciones, los diputados electos eran del partido mayoritario que iba en primer término en la fórmula (en este caso del PRI), todos sus diputados van para este bloque, pero no representan escaños para los demás coaligantes.

Fuente: elaboración propia, con información de la CEE $(2007,187 ; 2004,99)$.

Por otra parte, en este primer trienio de la legislatura local de 42 diputados (tanto de mayoría como de representación proporcional), el bloque priista tenía la mayoría absoluta (50 por ciento+1) de los escaños legislativos, sin embargo, la perdió estrepitosamente frente 
al PAN en 2006, que logró la mayoría en esta ocasión (incluso frente a la que ganó el PRI en la elección anterior). En la figura 3 se aprecia que en los comicios de 2003 el PAN tenía 11 diputados y 22 en los de 2006; mientras que el PRI pasó de 26 a 15.

El PRI conservó la mayor parte de los municipios de la entidad, perdió ocho de 51, pero no pudo retener la capital, Monterrey, que había obtenido en 2003, y conquistar otros municipios, bastiones tradicionales del Pan, como San Pedro Garza García, San Nicolás de los Garza y Santa Catarina, integrantes del área metropolitana de Monterrey (AMM) (véase figura 4). El PRI tenía el control de 40 municipios, de 51 que componen el estado, y en las elecciones de 2006 tenía 32. El PAN pasó de controlar 9 a 16 municipios.

\section{Figura 4}

Comparativo de municipios ganados por partido o coalición en Nuevo León, 2003 y 2006

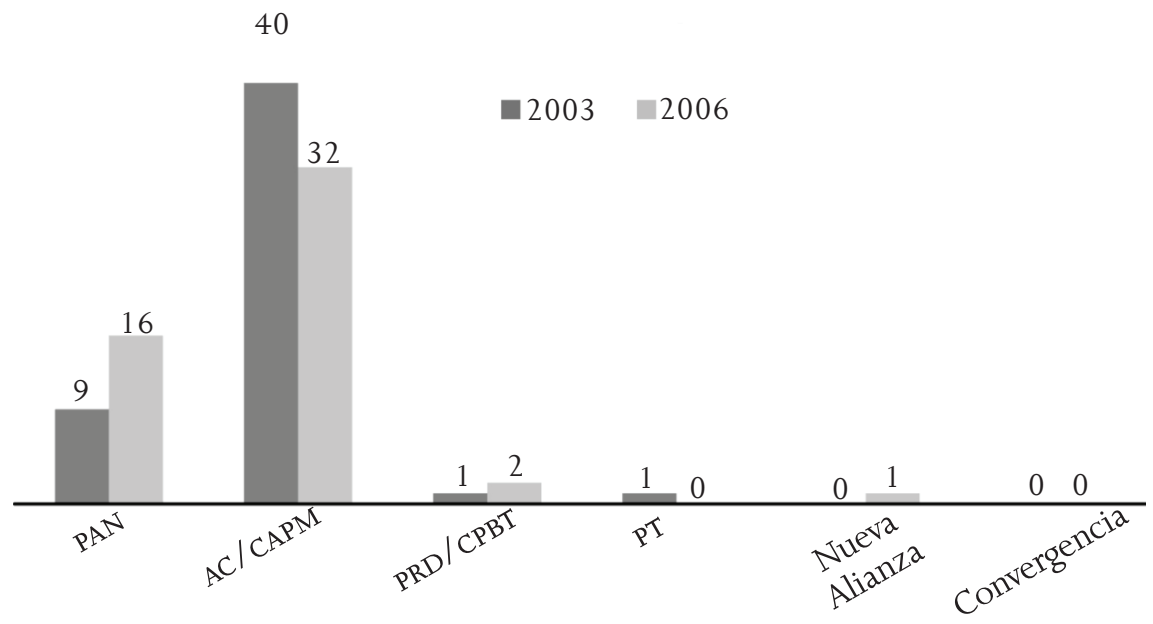

Nota: en 2003, el PRI estuvo en la coalición AC, con el PVEM, PFC, y el PLM. En el muncipio de Monterrey se agregó al PT. En 2006, el PRI contendió con la CAPM, con el PVEM. El PRD sólo en 2006 estuvo en la CPBT, con el PT.

Fuente: elaboración propia, con información de la CEE (2007; 2004). 
El PRI retuvo la gubernatura en las elecciones para gobernador en 2009; en coalición con partidos minoritarios, aunque éstos no hayan ganado más que la conservación de su registro. La coalición del PRI, Juntos por Nuevo León, logró 49 por ciento (859 442 votos) de la votación frente al principal opositor (el PAN), que obtuvo 43 (760 745 votos); la diferencia fue de 5.6 por ciento, nada comparable con la de 2003, en la que el PRI logró 22.86 (véase figura 5). En esta elección, el tema central de las campañas, posicionado por la agenda setting, fue la seguridad pública. "Daría mi vida por Nuevo León” fue el eslogan principal de campaña del candidato priista, cuyo objetivo-meta era comunicar y convencer a la ciudadanía que de resultar elegido tendría la vocación para devolverle la seguridad pública a Nuevo León. En su plataforma político-electoral fue identificado por una coyuntura urgente que resolver para la gobernabilidad del estado (Plataforma electoral 2009-2015).

\section{Figura 5}

Votación por partido o coalición

para la elección de gobernador en Nuevo León, 2009

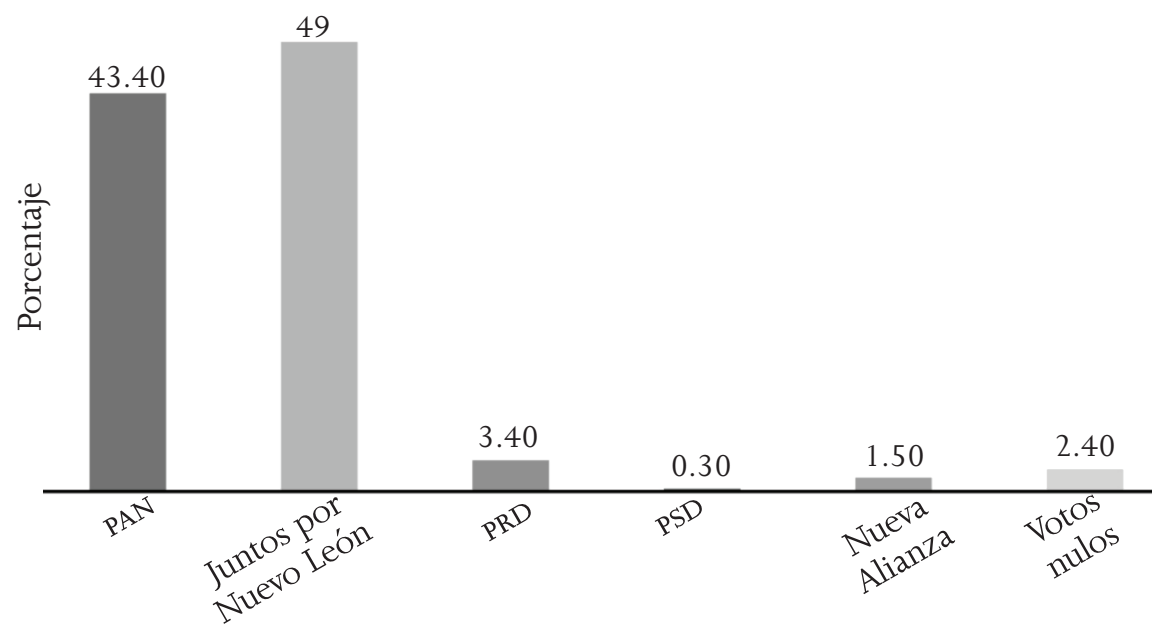

Nota: Coalición Juntos por Nuevo León (PRI, PVEM, Partido Cruzada Ciudadana, Partido Demócrata).

Fuente: elaboración propia, con información de la CEE $(2010,256)$. 
A pesar de que el PRI perdió espacios legislativos y algunas alcaldías, retuvo la gubernatura siguiente en el contexto de violencia, que incluso menguó durante las campañas (Medellín y Murillo 2010). El problema central que se intenta explorar aquí es cómo puede explicarse esta derrota electoral a mitad del sexenio, y luego retener el gobierno en la elección subsecuente. Este artículo sugiere relacionar esta pérdida de capital político con la seguridad pública. Sin embargo, este factor no fue determinante en el momento de la sucesión por la gubernatura, a pesar de algunas cifras negativas. Con la incidencia delictiva que se expone a continuación se comprenderá la crisis de seguridad, que antes no se había presentado de forma tan alarmante.

\section{El crecimiento del fenómeno delictivo.}

\section{Datos para entender la crisis}

Minowa (2011, 31-32) hace un recuento de las políticas de seguridad pública desde el sexenio de Martínez Domínguez (1979-1985) a partir del enfoque de la responsivess, resultado de un proceso de democratización. Una de sus conclusiones fue que en la administración de González Parás se incrementaron las políticas de seguridad pública, sobre todo en el ámbito de la prevención, pero aumentó la percepción de inseguridad por parte del ciudadano.

En el régimen de González Parás se alimentaron muchas esperanzas, en virtud de su formación profesional y expertise político. Si bien hubo beneficios en materia de obra pública y trasporte colectivo, como la construcción de la línea 2 del metro, del Hospital Materno Infantil y el emblemático desarrollo turístico paseo Santa Lucía, que une a la Macroplaza con el parque Fundidora. Además, uno de los más importantes fue posicionar a Monterrey como la Ciudad del Conocimiento (Gobierno del Estado de Nuevo León 2009). No obstante, el tema de la creciente inseguridad pública fue la principal demanda reconocida por el gobierno durante la campaña (González 2009) y en el Plan Estatal de Desarrollo (2003-2009).

Fue en la segunda parte de dicha administración cuando los indicadores se dispararon. De acuerdo con los especialistas, el detonante fue que los principales cárteles de la droga ubicaron a Nuevo León 
como espacio de lucha fratricida para la venta y el trasiego de estupefacientes, que marcó el signo de la violencia desde la guerra declarada por Felipe Calderón para combatirlos, en 2006 (Palacios 2009; Contreras y Zamora 2013). Años después, González Parás reconoció que el cambio súbito del contexto de seguridad pública en la entidad fueron los delitos relacionados con el narcotráfico, que se incrementaron en 2005, con la detención de José Luis Carrizales Coronado, alias El Tubi, un jefe de plaza del cártel de Sinaloa, en un restaurante al sur de la ciudad (González 2012). En los meses posteriores a esa detención hubo una ola de ejecuciones que tuvieron una gran repercusión en la opinión pública. De acuerdo con versiones periodísticas de la época, el incremento de la violencia se atribuyó a la pugna de la "plaza" entre los cárteles de Sinaloa y del Golfo (Astorga 2007, 234; Ravelo 2008, 31). La escisión del brazo armado del cártel del Golfo, -los Zetas- y su crecimiento como grupo autónomo derivó en una mayor disputa por el control territorial. Desde mayo de 2006 con frecuencia aparecieron, ante la opinión pública, noticias de ejecuciones de narcos, policías "madrinas" o protectores de algún cártel (Ravelo 2008, 32). Como señala este último autor, el asesinato que causó mayor revuelo político fue el de Marcelo Garza y Garza, en 2006, director de la recién estrenada Agencia Estatal de Investigaciones. Por lo menos desde el sexenio anterior ya había indicios de la presencia del crimen organizado en el estado, sin embargo la exacerbación de los delitos y la violencia sí corresponden mayormente al de González Parás (Medellín 2011).

Como puede medirse con los datos del régimen panista previo, en el mandato de González Parás las incidencias de alto impacto: robo, homicidios dolosos, extorsiones y secuestro fueron más. ${ }^{2}$ En términos comparativos, la tasa de robos con y sin violencia de 1998 a 2003, correspondiente al periodo del gobernador Canales Clariond, fue de 132 239, es decir, 574 por cada cien mil habitantes. En la gestión si-

2 Arango $(2009,14)$ advierte sobre el subregistro o cifra negra que en los datos oficiales no son tomados en cuenta, por tanto la necesidad de "desconfiar la veracidad de estos datos"; Barón (2012) también señala la precaución de no observar la impunidad delictiva, en donde la mayor parte de los delitos quedan sin sentencia condenatoria. Por supuesto, aquí no se desconoce esta debilidad de trabajar con datos oficiales, sin embargo, se considera que aún se pueden usar para notar las curvas de crecimiento del delito que sí se observan a pesar del subregistro. 


\section{Figura 6}

Comparativo de tasa de robo y homicidio en dos sexenios (por cada 100 mil habitantes)

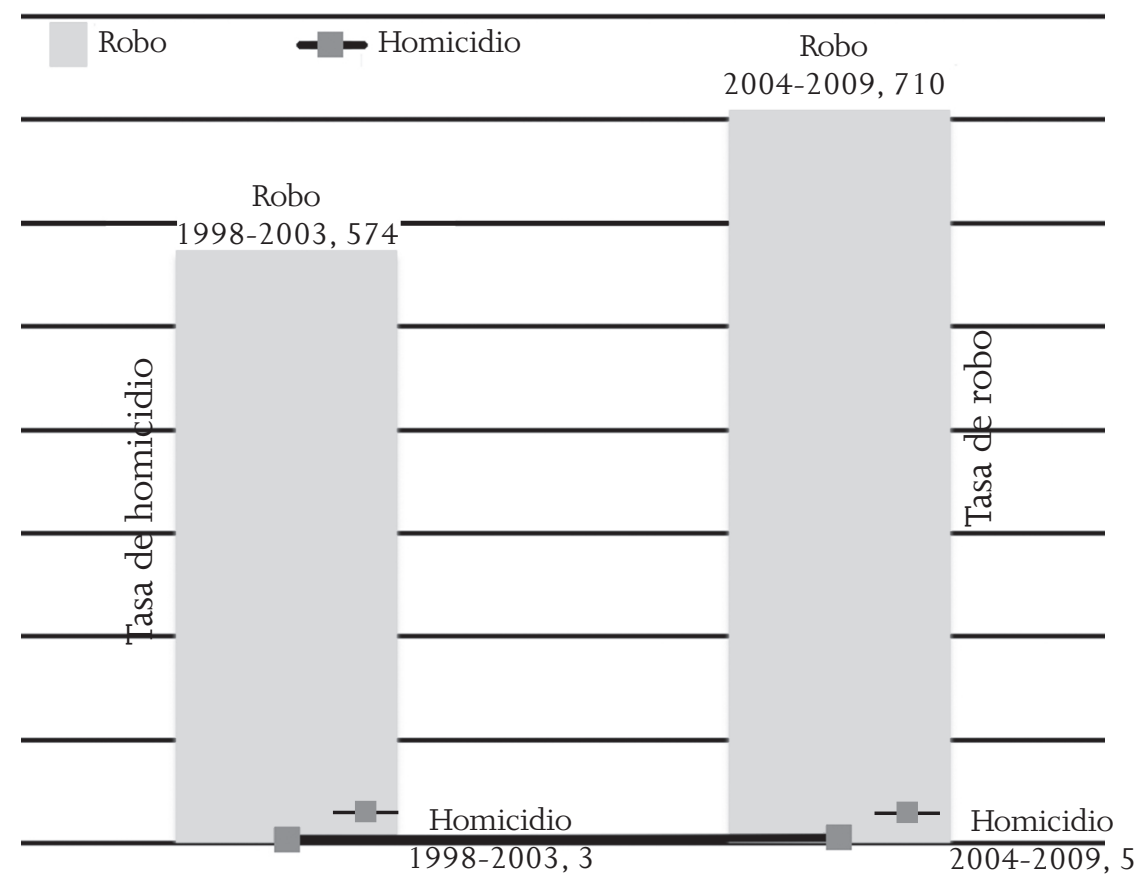

Nota: para las cifras de 1998-2003 se usó el censo del INEGI (2000) y para el siguiente periodo el censo del INEGi (2005).

Fuente: elaboración propia, con base en denuncias ante agencias del Ministerio Público de la Procuraduría General de Justicia de Nuevo León, reportadas ante al Sistema Nacional de Seguridad Pública durante ambos periodos (Secretaría de Gobernación 2009).

guiente la cifra se elevó a 178 872, lo que representa una tasa de 710 robos por cada cien mil habitantes. En lo que respecta a homicidios dolosos, la cifra también es elevada; en el sexenio anterior hubo tres por cada cien mil habitantes (813 en total); en el de González Parás fueron cinco por cada cien mil habitantes (1 253 en total).

En cuanto a las extorsiones y el secuestro, también fueron más en el sexenio de González Parás. Estos son los datos que menos reportes tienen ante la Procuraduría del estado. Por tanto, la cifra negra del delito debe ser mayor porque estos ilícitos no son denunciados. No obstan- 
te, de acuerdo con las cifras disponibles, puede señalarse que por cada cien mil habitantes la tasa de denuncia de secuestro en el sexenio de Canales fue de 0.127 , frente al de González Parás, de 0.150; en extorsiones fue de 0.685 a 1.382 respectivamente.

\section{Figura 7}

Comparativo de denuncias de extorsiones y secuestros en dos sexenios (tasa por cada 100 mil habitantes)

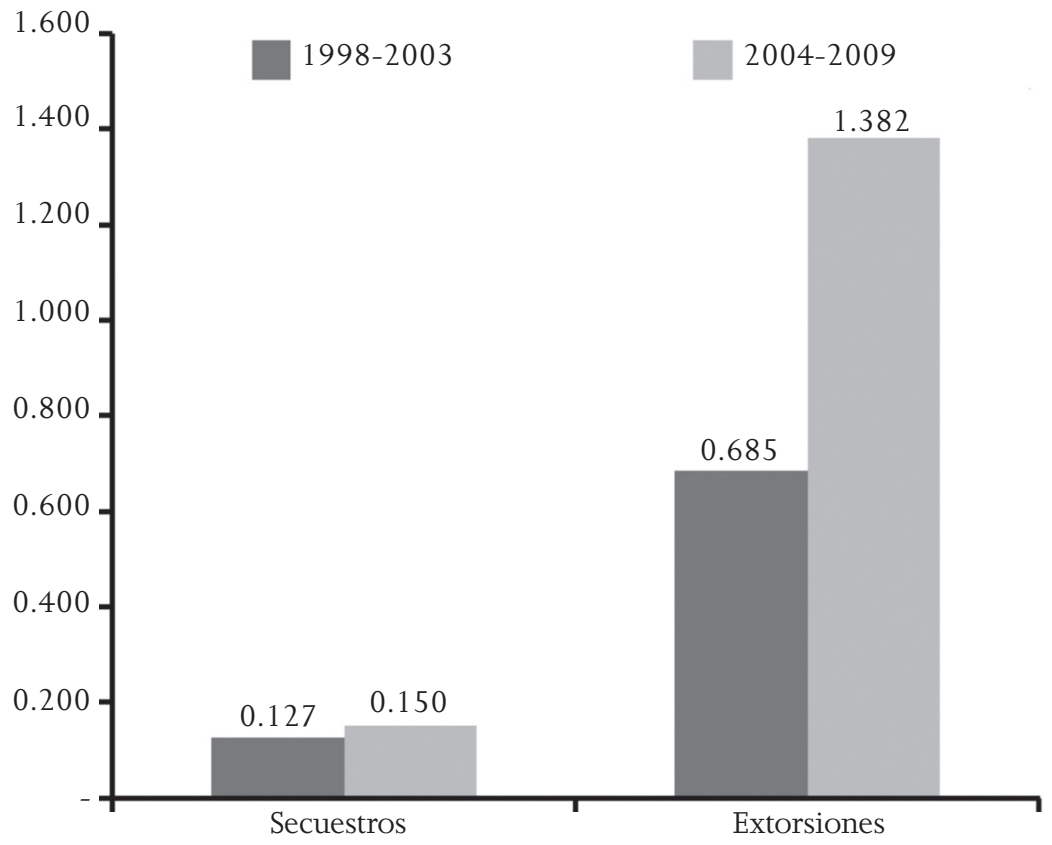

Nota: para las cifras de 1998-2003 se usó el censo del INEGI (2000), y para el siguiente periodo las del INEGI (2005).

Fuente: elaboración propia, con base en denuncias ante agencias del Ministerio Público de la Procuraduría General de Justicia de Nuevo León, reportadas ante el Sistema Nacional de Seguridad Pública durante ambos periodos (Secretaría de Gobernación 2009).

De acuerdo con los informes de gobierno del mandato de González Parás hubo 175217 robos; 1253 homicidios y 400 relacionados con el narcotráfico. Conforme a la tasa de crecimiento anual, la incidencia delictiva comenzó a crecer en 2005, sobre todo la de homi- 
cidios (143), que sumaron 22.2 por ciento, comparada con el dato anterior (117). También aumentaron los relacionados con el narcotráfico (106.7 por ciento); pasaron de 15 a 31. En 2006, el año de las elecciones intermedias, prácticamente todos los índices se elevaron: robos, 6 por ciento (24 602); homicidios, 25.9 (180) y los relacionados con el narcotráfico, 77.4 (55). Para el año siguiente todas las cifras siguieron creciendo: robos, 34.3 (33 043); homicidios, 57.2 (283) y las del narco, 130.9 (127). Sólo en 2008 hubo una tasa de reducción significativa en homicidios dolosos, -7.1 por ciento (263) y los relacionados con el narcotráfico, -40.9 (75) (véase figura 8). Para el cierre del sexenio, la única cifra alta fue la de los delitos vinculados con el narcotráfico, 29.3 por ciento (97); rubro en el que se reconocieron 400 muertes oficiales.

\section{Figura 8}

Tasa de crecimiento anual de robos, homicidios y los relacionados con el narcotráfico en el sexenio de González Parás

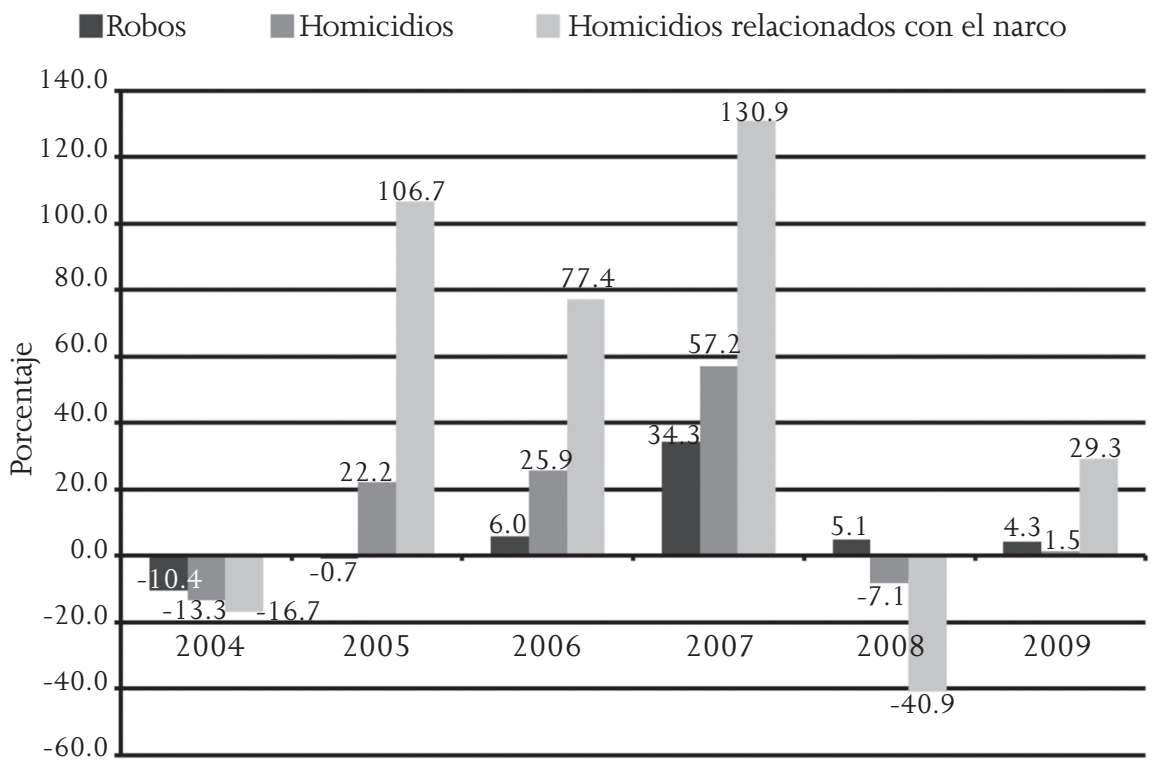

Fuente: elaboración propia, con base en denuncias ante agencias del Ministerio Público de la Procuraduría General de Justicia de Nuevo León, reportadas ante el Secretariado Ejecutivo del Sistema Nacional de Seguridad Pública (Secretaría de Gobernación 2009), y con información de la Procuraduría General de Justicia de Nuevo León (2011), para las cifras específicas de narcotráfico. 


\section{Leyes y políticas públicas, respuestas al reclamo}

En el Plan Estatal de Desarrollo (2004-2009, 39-41) un primer diagnóstico reconocía que el estado estaba asediado por la delincuencia y el crimen organizado; se admitía tener instituciones policiacas viciadas por la corrupción, policías insuficientes y escasa confianza ciudadana en las instituciones lo que limitaba las tareas de prevención del delito. Aunque González Parás en un principio rechazó la presencia del Ejército en territorio de Nuevo León, al final la aceptó, pero se apresuró a precisar que eso no significaba la militarización de la entidad (Astorga 2007, 237).

En las acciones específicas del gobierno estatal, las respuestas se basaron en tres apartados: normatividad jurídica, desarrollo institucional e instancias de participación ciudadana (véase figura 9). En primer lugar, se considera que la promulgación de la Ley Orgánica de la Administración Pública del Estado de Nuevo León marca una diferencia sustantiva en la organización del régimen de la coalición dominante; decretada al inicio del sexenio (octubre). Lo más destacable es que le dio un rango de secretaría, es decir la autonomía, a lo que antes, en la organización del Poder Ejecutivo, aparecía como subsecretaría dependiente de la Secretaría General de Gobierno.

En 2004, la nueva Ley Orgánica de la Procuraduría General de Justicia del Estado de Nuevo León dio la pauta para el nacimiento de la Agencia Estatal de Investigaciones, le otorgó la profesionalización a lo que se conoció como Policía Judicial, penetrada por el narcotráfico y la corrupción. Sin embargo, su primer director en funciones fue asesinado por el crimen organizado. Por otra parte, casi al final de su mandato, en 2008, se decretó la Ley de Seguridad Pública, la que tuvo mayor repercusión en la creación de instituciones e instancias de participación ciudadana, que otorgaron más infraestructura en la organización del estado, para salvaguardar este punto crítico de su administración. Se creó el Instituto Estatal de Seguridad Pública, el Centro de Cómputo, Comando, Control y Comunicaciones (C5), la Academia Estatal de Policía y el Consejo de Coordinación del Sistema Integral de Seguridad Pública del Estado, cuyas atribuciones serían el reclutamiento y la profesionalización de la seguridad pública, de acuerdo con estándares internacionales. Además, se puso énfasis 
en la prevención del delito, con programas muy específicos para su operatividad. En el Diario de debates, donde se aprobó esta iniciativa, se precisa su fundamentación:

En los últimos tiempos, hemos sido testigos de acontecimientos que han lesionado y trastocado la seguridad pública [...] generándose un sentimiento de temor y de angustia ante lo que se percibe no sólo como un incremento del fenómeno delictivo, sino también de mayores signos de violencia, presencia del crimen organizado y una considerable sensación ciudadana de inseguridad [...]" (Congreso del Estado de Nuevo León 2008, 86).

Esta ley se aprobó por unanimidad en el Congreso, y respaldada por 19 organizaciones civiles en busca de "[...] medidas innovadoras que contribuyan decididamente a generar un entorno donde impere el orden y la seguridad" (Congreso del Estado de Nuevo León 2008, 87). En ese sentido, se concuerda con García $(2011,266)$ cuando señala que esta ley estatal incluye por primera vez: “[...] un enfoque de seguridad pública basado en la modificación del ambiente físico para dificultar las manifestaciones de delitos, así como reducir su incidencia”. Contiene instancias de participación ciudadana importantes como un consejo ciudadano que estaría integrado, según el artículo 100, por padres de familia; representantes de organizaciones vecinales, sindicales, empresariales y de la sociedad civil; asociaciones de profesionistas y académicos, que serían convocados y elegidos por el Congreso (Periódico Oficial del Estado de Nuevo León 2008). Además, en su artículo 115 contiene los Comités de Participación Comunitaria que se integran " [...] por vecinos y organizaciones comunitarias para promover [...] la prevención comunitaria del delito y fomentar la cultura de la legalidad [...]" (Periódico Oficial del Estado de Nuevo León 2008, 58).

Las reformas al Código Penal, en 2004 y en 2007, obedecieron a la necesidad de homologar a la legislación local, la introducción del nuevo sistema penal acusatorio que agilizaría el "retraso en la resolución de los procedimientos además de que ofrecería salidas alternas a las sentencias judiciales" (Ayala et al. 2008, 20).

También se creó la ley y el respectivo Consejo de Valores y Cultura de la Legalidad, con el fin de "promover en el Estado de Nuevo León 
los valores universales y trascendentes del ser humano, así como la promoción de una cultura de la legalidad que robustezca el Estado de Derecho y el respeto a las reglas de convivencia armónica en la sociedad” (Periódico Oficial del Estado de Nuevo León 2007b) (véase figura 9).

\section{Figura 9}

Normas jurídicas y políticas públicas principales en materia de seguridad pública en el sexenio de González Parás

\begin{tabular}{|c|c|c|}
\hline $\begin{array}{l}\text { Norma jurídica } \\
\text { de origen }\end{array}$ & $\begin{array}{l}\text { Creación institucional } \\
\text { dependiente }\end{array}$ & $\begin{array}{l}\text { Instancias de participación } \\
\text { ciudadana dependiente }\end{array}$ \\
\hline $\begin{array}{c}\text { Ley Orgánica de la } \\
\text { Administración Pública } \\
\text { para el Estado de Nuevo } \\
\text { León (2003) }\end{array}$ & $\begin{array}{c}\text { Secretaría de } \\
\text { Seguridad Pública }\end{array}$ & \\
\hline $\begin{array}{c}\text { Ley Orgánica de la } \\
\text { Procuraduría General } \\
\text { de Justicia del Estado de } \\
\text { Nuevo León (2004) }\end{array}$ & $\begin{array}{c}\text { Agencia Estatal } \\
\text { de Investigaciones }\end{array}$ & \\
\hline $\begin{array}{l}\text { Ley de Seguridad Pública } \\
\qquad(2008)\end{array}$ & $\begin{array}{l}\text { Instituto Estatal de } \\
\text { Seguridad Pública } \\
\text { Centro de Cómputo, } \\
\text { Comando, Control y } \\
\text { Comunicaciones (C5) } \\
\text { Academia Estatal de } \\
\text { Seguridad Pública } \\
\text { Consejo de Coordinación } \\
\text { del Sistema Estatal de } \\
\text { Seguridad Pública }\end{array}$ & $\begin{array}{c}\text { Consejo Ciudadano } \\
\text { Comités de Participación } \\
\text { Comunitaria }\end{array}$ \\
\hline $\begin{array}{l}\text { Reformas al Código } \\
\text { Penal y al Código de } \\
\text { Procedimientos Penales } \\
\text { para el Estado de Nuevo } \\
\text { León }(2004,2007)\end{array}$ & $\begin{array}{c}\text { Adecuación del Poder } \\
\text { Judicial al nuevo sistema } \\
\text { penal acusatorio }\end{array}$ & \\
\hline $\begin{array}{c}\text { Ley para la Promoción de } \\
\text { Valores y Cultura de la } \\
\text { Legalidad (2007) }\end{array}$ & & $\begin{array}{l}\text { Consejo Estatal para la } \\
\text { Promoción de Valores y } \\
\text { Cultura de la Legalidad }\end{array}$ \\
\hline
\end{tabular}

Fuente: elaboración propia, con información del Periódico Oficial del Estado de Nuevo León (2008, 20 108; 2007a, 5-7; 2007b, 8-14; 2004a, 11-94; 2004b, 17-106; 2003, 7-60). 
En la figura 9 se observa que la Ley Orgánica de la Administración Pública, la Ley Orgánica de la Procuraduría General de Justicia del Estado y la reforma al Código Penal se promulgaron en el periodo legislativo de la mayoría absoluta priista (2003-2006). El resto se hizo cuando se perdió esta mayoría, no obstante, se sacaron con la cooperación del resto de las bancadas parlamentarias, que para efectos políticos el apoyo de la panista fue la más importante.

\section{Percepción de inseguridad ciudadana}

Con base en la encuestas de victimización, se ubicó el grado de percepción de confianza en la ciudadanía respecto a la seguridad en Nuevo León. La percepción de la inseguridad ciudadana por la presencia activa del narcotráfico fue un dato contundente, como lo refleja una encuesta en el AMM, dada a conocer un año después del término del sexenio referido, en el otoño de 2010. Por lo menos 50 por ciento de la población consideraba que su ciudad era muy insegura, y entre las razones expuestas sobresalían dos: 42 por ciento creía que se debía a la presencia del crimen organizado y el tráfico de drogas y 25 a que los organismos de seguridad no eran confiables (Centro de Investigaciones Económicas 2010, 35).

Por otra parte, la encuesta del Instituto Ciudadano de Estudios sobre la Inseguridad también arrojó datos similares sobre esta percepción de inseguridad. Los porcentajes se incrementaban de forma sostenida conforme avanzaba el sexenio. En la encuesta de 2004, 41 por ciento de la población creía que el estado era inseguro; para 2007 aumentó a 64, y en 2008 creció a 70 , y cerró con una cifra histórica de 82 , en 2009. En términos prácticos, ocho de cada diez nuevoleoneses consideraba inseguro a su estado al finalizar este sexenio.

En este panorama de desconfianza, hubo acciones de la sociedad civil para recuperar la seguridad, se convocaron marchas y movilizaciones por diversos actores en el AMM, fueron por lo menos cinco en cuatro meses, en 2008 (Contreras y Zamora 2013, 133). Pero también en otro estudio, Durin (2012) relató que en este periodo ocurrió el "exilio dorado" a Texas de numerosas familias regiomontanas de altos ingresos, para huir de la "guerra" en Nuevo León. 


\section{Figura 10}

Población de 18 años y más que considera que su estado es inseguro. Datos para Nuevo León

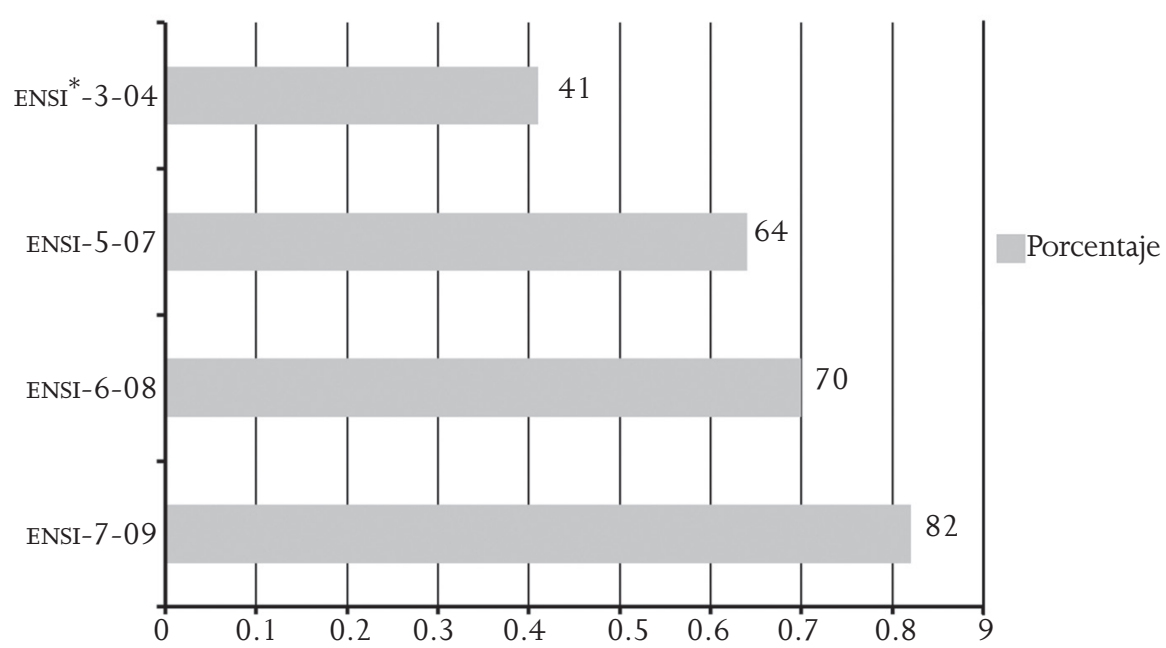

Nota: los datos de 2005 y 2006 no fueron publicados por el Instituto Ciudadano de Estudios sobre Inseguridad (ICESI).

* Encuesta nacional sobre inseguridad.

Fuente: elaboración propia, con datos del ICESI (2009).

\section{Conclusiones}

El efecto de los resultados electorales fue contrastante. Por un lado, el PRI en coalición mostró una disminución de los diputados locales y del número de municipios ganados en 2006; no obstante, obtuvo la gubernatura en 2009. ¿Cómo se deben interpretar estos resultados contrastantes? En un primer plano de análisis, la retención de la gubernatura no fue de manera holgada; González Parás ganó con un margen de 56.6 por ciento, frente al PAN, que obtuvo 33.8. El siguiente candidato priista, Rodrigo Medina de la Cruz, logró la victoria con 49 por ciento frente a 43.4 del PAN; es decir, perdieron 8 por ciento del electorado entre cada elección. Se gana, pero con un margen menor. 
En este artículo se asoció principalmente la estadística de incidencia delictiva, que tuvo un crecimiento exponencial a mitad del sexenio, con los resultados electorales de 2006. Se destacó que la tasa de crecimiento anual de los robos, los homicidios y los relacionados con el narcotráfico fue alta entre 2006 y 2007, aunque hubo un decremento en 2008 y 2009, excepto por las cifras del narcotráfico. El gobierno de González Parás admitió la presencia del crimen organizado desde el inicio de su gestión, y procuró acciones para la provisión del bien político de la seguridad. Para atender esto, se generó una reconfiguración institucional con la creación de la Secretaría de Seguridad Pública, en 2003. No obstante, fue hasta finales de 2008 cuando se decretó la Ley de Seguridad Pública, es decir, un año antes de que terminara su mandato. Este dato no sería importante a no ser porque a partir de esta ley se institucionalizó el andamiaje jurídico de nuevas instituciones en materia de seguridad pública, que serían el sello distintivo de esta administración. Estos bienes políticos de la seguridad (normas jurídicas y políticas públicas) evidencian la necesidad de la elite dominante priista de legitimarse, ante una emergencia en materia de seguridad pública. El triunfo de 2009 les permitió conservar su capital político, pero en la ruta de interpretación hay un resultado dual: mientras que en parte se cumplió con el objetivo de reducir los índices delictivos, a partir de 2008, los de percepción de inseguridad crecieron y se sostuvieron en el sexenio, de acuerdo con las cifras disponibles: 23 puntos porcentuales entre 2004 y 2007; 5 entre 2007 y 2008 y 12 para terminar 2009. Por tanto, la conclusión es que estos dos indicadores: la alta percepción de inseguridad ciudadana y las cifras de ejecuciones en narcotráfico, no tienen un efecto determinante para un resultado electoral adverso. La explicación de la pérdida de capital político a mitad del sexenio puede correlacionarse con el incremento del delito en 2006, pero se pone en duda que el tema de seguridad fuera decisivo para la elección de gobernador. Por tanto, hay dimensiones no exploradas aquí que pueden servir como líneas de investigación para ofrecer interpretaciones sobre el contexto del triunfo de 2009; estarían relacionadas con las fallas en la campaña del partido opositor, la escisión interna del panismo estatal o la sombra negra del nacional, debido a la experiencia fatídica en el ámbito federal (Felipe Calderón) o local (Fernando 
Canales Clariond). También puede hacerse un estudio específico de comportamiento electoral, donde se analice cómo se procesaron los temas de campaña en el votante (por rangos de edad, ocupación), y encontrar el linkage más fino asociado entre agenda setting e intención de voto. Otra dimensión podría estar relacionada con las ventajas competitivas que se le dieron al partido gobernante y la debilidad de los órganos electorales locales, lo que fue abordado desde la teoría de la calidad de la democracia (Medellín 2012). Este último punto enmarca que el proceso de campaña fue catalogado con una calidad democrática débil, lo que ensombrece la posibilidad de encontrar resultados genuinos para determinar la confiabilidad en la intención del votante promedio, ya que necesariamente tendrían que estar correlacionados con las variables del voto corporativo y el clientelismo electoral, que son las explicativas, muy determinantes en una victoria típica de partido dominante, por lo menos dada la historia de los procesos electorales en México. Es por eso que el presente estudio tomó el análisis por una vía alterna: revisar los datos de percepción de inseguridad ciudadana y de incidencia delictiva, para configurar el estado de la cuestión en materia de seguridad pública.

A pesar de este objetivo limitado, ya que sólo se prepondera la seguridad pública, como un posible efecto en los resultados electorales, se puede resumir que se encontraron efectos contrastantes: a mitad del sexenio priista se observó esta curva debilitante en su capital político, en el contexto de altos índices delictivos, pero no lo suficientemente determinante como para impedir su hegemonía y retención de la gubernatura.

Recibido en junio de 2014 Aceptado en noviembre de 2014

\section{Bibliografía}

Alcocer Villanueva, Jorge y José Luis Vázquez Alfaro. 2005. El impacto social de la percepción pública en México. En Seguridad pública.Voces diversas en un enfoque multidisciplinario, coordinado por Pedro José Peñaloza, 17-30. México: Porrúa. 
Arango, Arturo. 2005. Información confiable. Los problemas de la información estadística. Militarización y seguridad. El Cotidiano XXIV (153): 13-28.

Astorga, Luis. 2007. Seguridad, traficantes y militares. México: Tusquets.

Ayala, María de Lourdes, Javier Carrasco Solís, Agustín Sauceda Rangel y Antonio Varas Villavicencio. 2008. La reforma al sistema de justicia penal en Nuevo León: sus principales tendencias (2004-2007). Monterrey: Renace.

Barrón Cruz, Martín Gabriel. 2012. Violencia y seguridad en México en el umbral del siglo xxi. México: Novum-Instituto Nacional de Ciencias Penales.

Berlanga Santos, José Luis y Carlos Javier Vázquez Ferrel. 2009. Bases teóricas del estudio sobre el perfil del electorado neolonés. En Perfil del electoral neolonés, coordinado por la CEE, 65-90. Monterrey: UANL, Escuela de Graduados en Administración Pública (EGAP)-Tecnológico de Monterrey, Universidad de Monterrey (UDEM), Universidad Regiomontana (UR).

Blais, André. 2006. What affects voter turnout? Annual Review of Political Sciencie (9):111-125.

Campbell, Angus, Phillip Converse, Warren Miller y Donald Stokes. 1960. The American voter. Chicago: Chicago University Press.

Cantú Escalante, Jesús y Claudia E. Garza Urquidi. 2009. El votante neolonés: el partidista, el personalista y el informe. En Perfil del elector neolonés, coordinado por la CEE, 91-118. Monterrey: UANL, EGAP, UDEM, UR.

Cavazos Garza, Israel e Isabel Ortega Ridaura. 2010. Nuevo León. Historia breve. México: Fondo de Cultura Económica (FCE).

Centro de Investigaciones Económicas. 2010. Primera encuesta de cultura ciudadana aplicada en el área metropolitana de Monterrey. Documento final. Monterrey: Facultad de Economía, UANL. 
CEE. 2010. Memoria y estadísticas. Informe del proceso electoral Nuevo León 2008 -2009. Monterrey: CEE.

CEE. 2007. Memoria y estadísticas. Informe del proceso electoral Nuevo León 2006. Monterrey: CEE.

CEE. 2004. Memoria y estadísticas. Informe del proceso electoral Nuevo León 2003. Monterrey: CEE.

Congreso del Estado de Nuevo León. 2008. Diario de debates. LXXI Legislatura 239-LXXI, primer periodo, año III, 17 de septiembre, Monterrey.

Contreras, Camilo y Gabriela Zamora. 2013. El espacio público en disputa: Monterrey, su pálida e incipiente sociedad civil. En Violencia $e$ inseguridad en los estados fronterizos del norte de México en la primera década del siglo XxI, coordinado por Vicente Sánchez Munguía, 119-198. México: Red Regional de Investigación en Seguridad Pública y Desarrollo Social.

Downs, Anthony. 1957. An economic theory of democracy. Nueva York: Harper Collins.

Durin, Séverine. 2012. Lo que la guerra desplazó: familias del noreste de México en el exilio. Desacatos (38): 29-42.

Garland, David. 2001. La cultura del control. Crimen y orden social en la sociedad contemporánea. Barcelona: Gedisa.

García Gómez, José. 2011 . Urbanismo y programas de seguridad pública en la frontera norte de México. Frontera Norte 23 (46): 263 268.

Gobierno del Estado de Nuevo León. 2009. Frentes de innovación estratégica. Monterrey: Instituto Nacional de Administración Pública, Fondo Editorial Nuevo León. 
González Parás, Natividad. 2012. La gobernabilidad democrática en México. Conferencia dictada en el auditorio del Centro de Investigaciones de T. Jurídica y Criminológica, Facultad de Derecho y Criminología, Monterrey, UANL, 5 de noviembre.

González Parás, Natividad. 2009. Visión, compromisos y resultados. Gestión gubernamental de José Natividad González Parás, Nuevo León, 2003-2009. Monterrey: Fondo Editorial Nuevo León.

Ibarra Palafox, Francisco. 2009. El leviatán encadenado o la legitimidad de la violencia estatal. En Uso legítimo de la fuerza, coordinado por Eduardo Medina Mora, 21-26. México: Instituto Nacional de Ciencias Penales.

ICESI. 2009. Sexta Encuesta nacional sobre inseguridad, ENSI-6. Resultados, primera parte, nacionales y por entidad federativa: http:// www.icesi.org.mx/documentos/encuestas/encuestasNacionales/ ENSI-6.pdf $>$ (6 de agosto de 2012).

INEGI. 2005. II Conteo de población y vivienda: http://www.inegi. org.mx/est/contenidos/proyectos/ccpv/cpv2005/ (20 de julio de 2013).

INEGI. 2000. XII Censo general de población y vivienda: http://www. inegi.org.mx/est/contenidos/Proyectos/ccpv/cpv2000/ (15 de julio de 2013).

Jiménez Ornelas, René Alejandro. 2005. La percepción, componente indispensable contra la inseguridad. En Seguridad pública. Voces diversas en un enfoque multidisciplinario, coordinado por Pedro José Peñaloza, 329-343. México: Porrúa.

Koschick, Murilo. 2004. Teorías del comportamiento electoral y algunas de sus aplicaciones. Revista Mexicana de Ciencias Políticas y Sociales XLVI (190): 47-72.

Lazarfeld, Paul, Bernard Berelson y Helen Guadet.1969. The people's choice. Nueva York: Columbia University. 
Mc Combs, Maxwell y Donald Shaw. 1972. The agenda setting function of mass media. The Public Opinion Quarterly 36 (2): 176-187.

Medellín Mendoza, Laura. 2012. La accountability del proceso electoral del 2009 en Nuevo León. Diagnóstico en el marco de la teoría sobre la calidad de la democracia. Revista Iberofórum vi (14):157-194.

Medellín Mendoza, Laura. 2011 . Liberalización y gobernabilidad en Nuevo León. Monterrey: UANL.

Medellín Mendoza, Laura y Jorge E. Murillo.2010. Un retrato de las elecciones locales y la espiral de violencia en Nuevo León. En Elecciones en tiempos de guerra. Baja California, Chihuahua, Durango, Nuevo León, Sinaloa,Tamaulipas, Veracruz, coordinado por Ernesto Hernández Norzagaray, 131-156. Culiacán: Universidad Autónoma de Sinaloa.

Minowa, Shigeru. 2011. Políticas de la seguridad pública en el estado de Nuevo León y responsiveness del gobierno después de la democratización. Conocimiento y Cultura Jurídica IV (9): 9-31.

Palacios Pámanes, Gerardo. 2009. Desafíos a la seguridad en Nuevo León. En Atlas de la seguridad y la defensa de México, editado por Raúl Benítez Manaut, 179-182. México: Colectivo de Análisis de la Seguridad con Democracia.

Peña Razo, Francisco Javier. 2011. La seguridad humana para la reconstrucción del concepto de seguridad pública en México. México: Miguel Ángel Porrúa, Universidad de Guadalajara.

Periódico Oficial del Estado de Nuevo León. 2008. Ley de Seguridad Pública para el Estado de Nuevo León. Decreto 279, número 127. 22 de septiembre.

Periódico Oficial del Estado de Nuevo León. 2007a. Reforma al Código de Procedimientos Penales del Estado de Nuevo León. Decreto 73, número 73.30 de marzo. 
Periódico Oficial del Estado de Nuevo León. 2007b. Ley Para la Promoción de Valores y Cultura de la Legalidad. Decreto 72, número 43. 23 de marzo.

Periódico Oficial del Estado de Nuevo León. 2004a. Ley Orgánica de la Procuraduría General de Justicia del Estado de Nuevo León. Decreto 115 , número 97.30 de julio.

Periódico Oficial del Estado de Nuevo León. 2004b. Reforma al Código Penal del Estado de Nuevo León. Decreto 118, número 96. 28 de julio.

Periódico Oficial del Estado de Nuevo León. 2003. Ley Orgánica de la Administración Pública para el Estado de Nuevo León. Decreto 8, número 131. 9 de octubre.

Plan Estatal de Desarrollo. 2004-2009. Por un gobierno humanista, democrático, competitivo y con resultados: http://www.nl.gob. $\mathrm{mx} /$ ?P=plan_desarrollo_historico (6 de mayo de 2012).

Plataforma electoral 2009-2015. Juntos por Nuevo León. Elección de gobernador. Monterrey: PRI.

Procuraduría General de Justicia de Nuevo León. 2011. Informe de homicidios dolosos relacionados con el narcotráfico (2004-2009). Dirección de Asuntos y Estudios Legislativos, oficio 11/2011, Monterrey. 1 de abril.

Ramírez Echeverri, Juan David. 2010. Thomas Hobbes y el Estado absoluto: del Estado de razón al Estado de terror. Medellín: Facultad de Derecho y Ciencias Políticas, Universidad de Antioquía.

Ramírez Saavedra, Beatriz Eugenia. 2010. La crisis de seguridad y la agenda de riesgos de seguridad nacional. México: Porrúa.

Ravelo, Ricardo. 2008. Herencia maldita. El reto de Calderón y el nuevo mapa del narcotráfico. México: Random House Mondadori. 
Robin, Corey. 2009. El miedo. Historia de una idea política. México: FCE.

Rojas, Javier. 2006. Fábricas e industria. Símbolos de la cultura industrial regiomontana. Monterrey: Instituto de Investigaciones Históricas de Nuevo León-Consejo para la Cultura y las Artes de Nuevo León.

Rotberg, Robert. 2007. El fracaso y el colapso de los Estados-nación. Descomposición, prevención y reparación. En Los Estados fallidos 0 fracasados: un debate inconcluso y sospechoso, coordinado por Robert I. Rotberg et. al, 151-237. Bogotá: Siglo del Hombre Ed. Universidad de los Andes, Pontificia Javeriana-Instituto Pensar.

Secretaría de Gobernación. 2009. Incidencia delictiva reportada ante el sistema nacional de seguridad pública: http://secretariadoejecutivo.gob.mx/en/SecretariadoEjecutivo/Incidencia_Delictiva_ Nacional_fuero_común> (6 de julio de 2012).

Tedesco, Laura. 2007. El Estado en América Latina ¿fallido o en proceso de formación? FRIDE working paper (37): //www.fride. org/publicacion/177/el-estado-en-america-latina:- ¿fallido-oen-proceso-de-formacion? (8 de agosto de 2012).

Yates, Arthur. 2014. The right to punish in Thomas Hobbe's Leviathan. Journal of the History of Philosophy 52 (2): 233-254. 
